\title{
Inferring symbolic dynamics of chaotic flows from persistence
}

\author{
Gökhan Yalnız ${ }^{1}$ and Nazmi Burak Budanur ${ }^{2}$ \\ 1) Physics Department, Boğaziçi University, 34342 Istanbul, Turkey a) \\ ${ }^{2)}$ Nonlinear Dynamics and Turbulence Group, IST Austria, 3400 Klosterneuburg, \\ Austria $^{\text {b) }}$
}

(Dated: 13 January 2020)

\begin{abstract}
We introduce "state space persistence analysis" for deducing the symbolic dynamics of time series data obtained from high-dimensional chaotic attractors. To this end, we adapt a topological data analysis technique known as persistent homology for the characterization of state space projections of chaotic trajectories and periodic orbits. By comparing the shapes along a chaotic trajectory to those of the periodic orbits, state space persistence analysis quantifies the shape similarity of chaotic trajectory segments and the periodic orbits. We demonstrate the method by applying it to the three-dimensional Rössler system and a thirty-dimensional discretization of the Kuramoto-Sivashinsky partial differential equation in $(1+1)$ dimensions.
\end{abstract}

Keywords: high-dimensional chaos, symbolic dynamics, topological data analysis, persistent homology

One way of studying chaotic attractors systematically is through their symbolic dynamics, in which one partitions the state space into qualitatively different regions and assigns a symbol to each such region. ${ }^{1-3}$ This yields a "coarse-grained" state space of the system, which can then be reduced to a Markov chain encoding all possible transitions between the states of the system. While it is possible to obtain the symbolic dynamics of low-dimensional chaotic systems with standard tools such as Poincaré maps, when applied to high-dimensional systems such as turbulent flows, these tools alone are not sufficient to determine symbolic dynamics. ${ }^{4,5}$ In this paper, we develop "state space persistence analysis" and demonstrate that it can be utilized to infer the symbolic dynamics in very high-dimensional settings.

\section{INTRODUCTION}

One of the defining features of chaos is the sensitive dependence on initial conditions, ${ }^{1,2,6}$ which is a statement of the exponential amplification of noise under chaotic dynamics. The practical corollary of this fundamental property is that any prediction based on integrating equations of motion of a chaotic system starting from an initial condition is exponentially wrong in time since all measurements come with noise. Thus, even with the advanced computing technologies of our day, the question of "What is the future state of a chaotic system based on a measurement of its current state?" can only be answered for a finite time horizon. A different and more tractable question is the following: What are the possible

\footnotetext{
a) Current address: IST Austria, 3400 Klosterneuburg, Austria

b)Electronic mail: burak.budanur@ist.ac.at
}

future states of a chaotic system given an approximate measurement of its current state?

The answer to this question begins with a "coarsegraining" of the system's state space into regions with qualitative differences, followed by determining the transition rules between these regions. The associated methods of the dynamical systems theory are known as symbolic dynamics. ${ }^{1-3}$ While these techniques lie at the heart of some of the most fundamental results of chaos theory such as Smale's proof ${ }^{7,8}$ of the Birkhoff-Smale theorem, ${ }^{9}$ existing symbolic dynamics methods can only be applied to low-dimensional systems, namely the ones that can be effectively described by one- or two-dimensional maps.

Some examples of continuous-time chaos, such as the Lorenz $^{10}$ and Rössler ${ }^{11}$ systems, can be reduced to onedimensional return maps by means of Poincaré sections. ${ }^{12,13}$ This is possible because both models are threedimensional with a single positive Lyapunov exponent, which yields a "thin" attractor due to strong contraction in the direction pointing outwards from the attractor. ${ }^{3,6}$ Many real-life examples of chaos, in contrast, take place in systems with many $(D \gg 3)$ degrees of freedom. Examples include fluid turbulence, ${ }^{14}$ cardiac dynamics, ${ }^{15}$ and evolution. ${ }^{16}$ Generally, such systems cannot be reduced to low-dimensional maps, except in special cases close to the onset of chaos. ${ }^{17}$ However, the observations based on computer simulations ${ }^{4,5,18}$ suggest that highdimensional systems such as turbulent flows exhibit a large catalog of motions that can be associated with the time-periodic solutions of the governing equations. While the methods for locating unstable periodic orbits of highdimensional dynamical systems are well developed, ${ }^{19}$ to the best of our knowledge, there exists no technique for the unsupervised identification of similarities between chaotic trajectory segments and periodic orbits of highdimensional systems. In this paper, we shall demonstrate that this can be achieved via topological data analysis.

Topological data analysis is an active field of research with a continuously growing domain of applications. ${ }^{20} \mathrm{In}$ a broad sense, topological data analysis methods aim to extract significant geometric features of high-dimensional 
and/or noisy data sets. Arguably the most popular tool in this field is persistent homology ${ }^{20,21}$ which was recently applied to various representations of data produced by dynamical systems. Some examples are physical space data obtained from biological aggregation mod$\mathrm{els}^{22}$ and fluid simulations, ${ }^{23}$ and time series data from chaotic systems. ${ }^{24,25}$ Differently from these examples, in the present work, we compute persistence in state space in order to compare the shapes of chaotic trajectory segments to those of periodic orbits in high-dimensional settings.

In this paper, we propose a novel technique for inferring the symbolic dynamics of chaotic motion in arbitrary dimensions. We name our method "state space persistence analysis", and illustrate its core ideas on the three-dimensional Rössler system. We then apply the method to the Kuramoto-Sivashinsky partial differential equation (PDE) and show that the system's spatiotemporally chaotic dynamics can be approximated by a Markov chain based on four distinct periodic orbits. The rest of the paper is organized as follows. In section II, we recapitulate the core concepts from the dynamical systems theory and topological data analysis, which form the foundations of state space persistence analysis. In section III, we lay out the steps of state space persistence analysis for a generic continuous-time dynamical system. We demonstrate our method with applications in section IV, discuss our results in section $\mathrm{V}$ and conclude in section VI.

\section{PRELIMINARIES}

We consider dynamical systems defined by a $D$ dimensional state space $\mathcal{M} \subset \mathbb{R}^{D}$ and a smooth flow map $f^{t}(\xi)$ that maps state vectors $\xi \in \mathcal{M}$ as

$$
\xi(t)=f^{t}(\xi(0)),
$$

where $t \in \mathbb{R}^{+}$is the time variable. Although it is not a general requirement, in the examples we consider, $f^{t}(\xi(0))$ is related to an ordinary differential equation (ODE)

$$
\dot{\xi}=v(\xi)
$$

through the relation

$$
f^{t}(\xi(0))=\xi(0)+\int_{0}^{t} v\left(\xi\left(t^{\prime}\right)\right) d t^{\prime},
$$

where $v(\xi)$ is called the state space velocity. While in the examples worked out here we always use the Euclidean inner product

$$
\left\langle\xi^{(i)}, \xi^{(j)}\right\rangle=\sum_{k=1}^{D} \xi_{k}^{(i)} \xi_{k}^{(j)},
$$

we expect that the topological methods we develop here do not depend strongly on the particular choice of norm. In (4), subscripts denote vector components and superscripts in parentheses denote labels.

\section{II.1. Symbolic dynamics and shadowing}

We assume that the state space $\mathcal{M}$ is coarse-grained into regions $\mathcal{M}^{(A)}, \mathcal{M}^{(B)}, \mathcal{M}^{(C)} \ldots$ such that a trajectory $\xi(t)$ for $t \in\left[0, t_{F}\right]$ can be associated with an itinerary $\sigma_{1} \sigma_{2} \sigma_{3} \ldots$ with $\sigma_{i} \in\{A, B, C, \ldots\}$ according to the successive state space regions visited by the trajectory. Further, we assume that the system admits periodic orbits such that every point $\xi^{(p)}$ on a periodic orbit $p$ satisfies

$$
\xi^{(p)}=f^{T_{p}}\left(\xi^{(p)}\right)
$$

for a nonzero period $T_{p}$ and its integer multiples. By definition, a periodic orbit has a cyclic itinerary, such as $\overline{\sigma_{1} \sigma_{2} \ldots \sigma_{n}}$, where the overline denotes infinite repetition. In what follows, we use the itinerary of a periodic orbit as its label when an itinerary is known. Finally, we assume that the first $n$ symbols in the itinerary of a trajectory $\xi(t)$ for $t \in\left[0, t_{F}\right]$ are the same with that of the periodic point $\xi^{(p)}$ if $\xi(0)$ and $\xi^{(p)}$ are sufficiently close in an appropriately defined state space distance measure. When a segment of an itinerary of a generic trajectory is the same with that of a periodic orbit, we say that "the trajectory 'shadows' the periodic orbit". Let us illustrate these concepts with an example.

The Rössler system is defined by the set of ODEs ${ }^{11}$

$$
\dot{x}=-(y+z), \quad \dot{y}=x+0.2 y, \quad \dot{z}=0.2+z(x-5.7) \text {. }
$$

The numerical integration of (6) reveals a chaotic attractor, which we visualize in Fig. 1 (a) by a trajectory on it. The temporal length of this trajectory is $t_{f}=2000$, which covers the attractor sufficiently for visualization purposes. We define a Poincaré section $\hat{\mathcal{M}}$ as the half hyperplane of points $\hat{\xi} \in \hat{\mathcal{M}}$ which satisfy

$$
\left\langle\hat{\xi}-\hat{\xi}^{\prime}, \eta\right\rangle=0 \quad \text { and } \quad\langle v(\hat{\xi}), \eta\rangle>0,
$$

where $\hat{\xi}^{\prime}$ and $\eta$ are called the "section template" and the "section normal", respectively. For the choices of $\hat{\xi}^{\prime}=$ $(0,-1,0)$ and $\eta=(1,0,0)$ we show the Poincaré section defined by (7) in Fig. 1 (a) as a transparent surface.

Let $\hat{\xi}[n]$ be a state vector on the Poincaré section (7) at the discrete time $n$. The Poincaré map is the discretetime system

$$
\hat{\xi}[n+1]=\mathcal{P}(\hat{\xi}[n])=f^{\Delta t_{n}}(\hat{\xi}),
$$

where $\Delta t_{n}$ is the "first-return time", that is, the minimum time required for the trajectory of $\hat{\xi}[n]$ to intersect the Poincaré section (7). As illustrated by Fig. 1 (a), the trajectories on the Rössler attractor intersect the Poincaré section (7) along what appears to be a one-dimensional curve. This suggests the arc length along this curve as a natural parametrization for the Poincaré map. We interpolate this curve with cubic splines and use the data to obtain the unimodal Poincaré return map shown in Fig. 1 (b). 

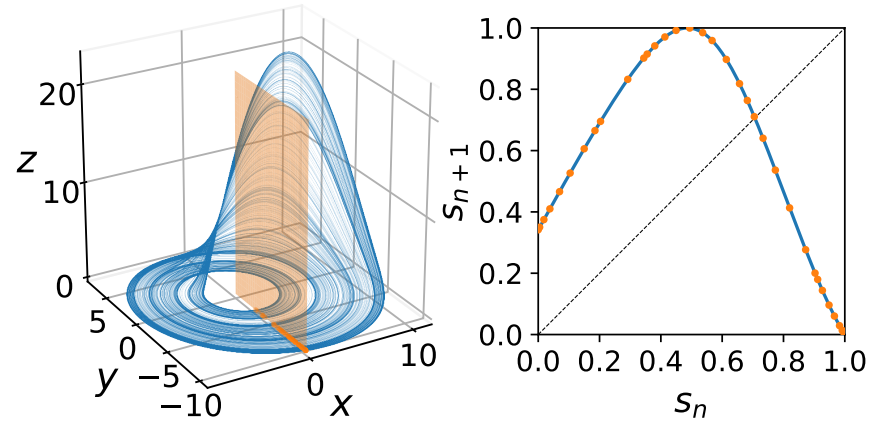

(a)

(b)

FIG. 1. (a) A trajectory (blue) on the Rössler attractor and its intersections (orange points) with the Poincaré section (7). The Poincaré section (7) is visualized as a transparent surface. (b) First-return data (orange) and the Poincaré map (blue), parameterized by the arc length along the interpolation of the intersections in (a). In both (a) and (b), every tenth intersection is shown for better visibility.

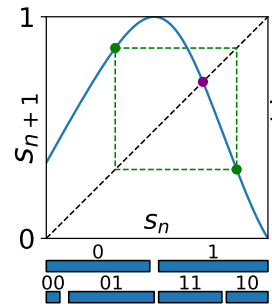

(a)

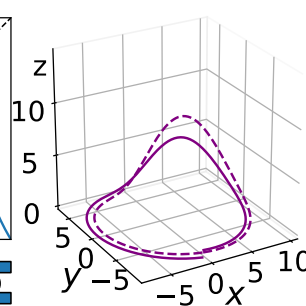

(b)

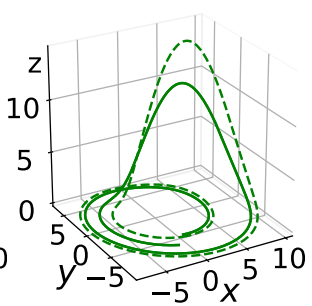

(c)
FIG. 2. (a) Periodic orbits $\overline{1}$ (purple point), $\overline{01}$ (green points, connected with dashed line segments), and the partitioning of the Rössler system's Poincaré map. (b) Periodic orbit $\overline{1}$ (purple, dashed) and a shadowing trajectory segment (purple, solid) of the Rössler system. (c) Periodic orbit $\overline{01}$ (green, dashed) and a shadowing trajectory segment (green, solid) of the Rössler system.

We are now in position to partition the state space of the Rössler system into regions. The return map of Fig. 1 (b) has one critical point $\hat{\xi}^{(c)} \approx 0.4868$, at which the derivative of the Poincare map is zero. Let us define regions $\hat{\mathcal{M}}^{(0)}$ and $\hat{\mathcal{M}}^{(1)}$ as

$$
\begin{aligned}
& \hat{\mathcal{M}}^{(0)}=\left\{\hat{\xi} \in \hat{\mathcal{M}} \mid \hat{\xi}<\hat{\xi}^{(c)}\right\}, \\
& \hat{\mathcal{M}}^{(1)}=\left\{\hat{\xi} \in \hat{\mathcal{M}} \mid \hat{\xi}>\hat{\xi}^{(c)}\right\} .
\end{aligned}
$$

With these definitions, we can now assign each trajectory on the Rössler attractor a binary symbol sequence. In particular, we can now enumerate the periodic orbits of the Rössler system with binary numbers. Fig. 2 (a) shows the two shortest periodic orbits $\overline{1}$ and $\overline{01}$ of the Rössler system on the Poincaré map and Fig. $2(\mathrm{~b}-\mathrm{c})$ shows these orbits in the full state space.

It is straightforward to confirm that a point on the Poincaré map Fig. 2 (a) that is close to a periodic orbit has initially the same itinerary as that of the periodic orbit. We show examples of such "shadowing" trajectories along with the periodic ones in Fig. $2(\mathrm{~b}-\mathrm{c})$. At the bottom of Fig. 2 (a), we show the subpartitioning of the unimodal map with respect to the itineraries of the points on it. This partitioning can be confirmed by inspection. For example, an initial condition picked from partition $\hat{\mathcal{M}}^{(10)}$ starts out at partition $\hat{\mathcal{M}}^{(1)}$ and lands at partition $\hat{\mathcal{M}}^{(0)}$ after one iteration of the Poincaré map. Further iterates of the map would result in finer partitions with longer and longer periodic orbits. For details, we refer the interested reader to Refs. 2 and 3.

The similarities of the periodic orbits and the shadowing trajectory segments in Fig. $2(\mathrm{~b}-\mathrm{c})$ constitute the key intuition of state space persistence analysis. In general, it is not possible to reduce the dynamics of a chaotic attractor into a unimodal map such as Fig. 1 (b). However, one can still find periodic orbits and compare the shapes of trajectory segments to those of the periodic orbits. Our next step is to introduce persistent homology which we utilize for this purpose.

\section{II.2. Persistent homology}

Persistent homology is a mathematical framework for extracting significant shapes in a data set. In this section, we illustrate the persistent homology concepts that we incorporate in our method through an example, while trying to avoid the technical language as much as possible. For in-depth introductions, we refer the reader to the survey $^{21}$ and the textbook ${ }^{26}$ by Edelsbrunner and Harer and the "roadmap" 27 by Otter et al.

In our applications, we consider a data set

$$
\Xi=\left\{\tilde{\xi}^{(1)}, \tilde{\xi}^{(2)}, \ldots, \tilde{\xi}^{(N)}\right\}
$$

that is composed of projections $\tilde{\xi}^{(i)}=\mathbf{P} \xi^{(i)}$ of state vectors $\xi^{(i)}$ sampled from a trajectory of a dynamical system. $\mathbf{P}$ is a projection operator specific to the application. Fig. 3 (a) shows an example of such a data set from the Rössler system as a projection onto the $(x, y)$ plane. These points are sampled from the periodic orbit $\overline{1}$ (Fig. 2) of the Rössler system with a constant time step of $t_{s}=0.45$.

For the analysis to follow, we need a distance function for the projected data set, which we define as

$$
d\left(\tilde{\xi}^{(i)}, \tilde{\xi}^{(j)}\right)=\left\langle\tilde{\xi}^{(i)}-\tilde{\xi}^{(j)}, \tilde{\xi}^{(i)}-\tilde{\xi}^{(j)}\right\rangle^{1 / 2} .
$$

Let $r \geq 0$ be the "resolution" (a distance parameter), $\Xi_{r}$ denote a continuous sequence of sets of subsets of $\Xi$ parameterized by $r$, and $\Xi_{0}=$ $\left\{\left\{\tilde{\xi}^{(1)}\right\},\left\{\tilde{\xi}^{(2)}\right\}, \ldots,\left\{\tilde{\xi}^{(N)}\right\}\right\}$. The sets $\Xi_{r}$ are formed by the union of $\Xi_{0}$ with all edges $\left\{\tilde{\xi}^{(i)}, \tilde{\xi}^{(j)}\right\}$ such that $d\left(\tilde{\xi}^{(i)}, \tilde{\xi}^{(j)}\right) \leq r$ and all triangles $\left\{\tilde{\xi}^{(i)}, \tilde{\xi}^{(j)}, \tilde{\xi}^{(k)}\right\}$ such that all pairwise distances $d\left(\tilde{\xi}^{(i, j, k)}, \tilde{\xi}^{(i, j, k)}\right) \leq r$. In general, this sequence is extended to include tetrahedrons and higher-dimensional generalizations. However, we stop at triangles since this is sufficient for our applications. We 


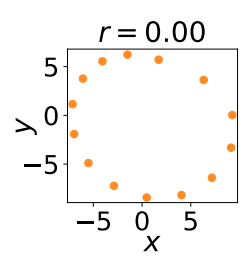

(a)

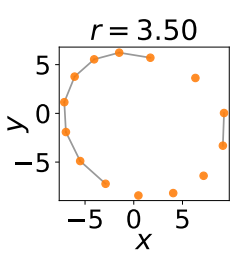

(b)

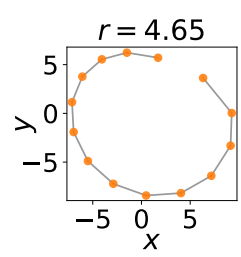

(c)

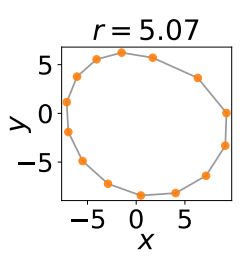

(d)

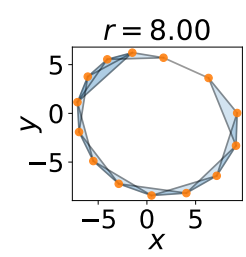

(e)

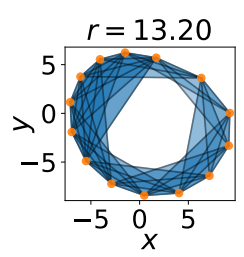

(f)

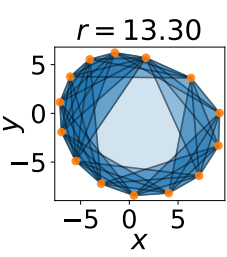

(g)

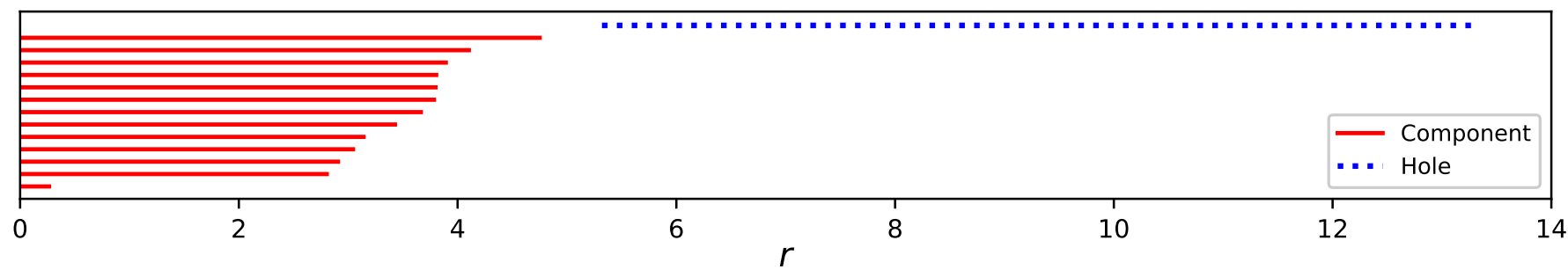

(h)

FIG. 3. (a-g) Visualizations of the sequence of sets $\Xi_{r}$ corresponding to a data set sampled from the periodic orbit $\overline{1}$ of the Rössler system. The parameter $r$ is a distance threshold that sets the connectivity of the points in the data set: Two points that are closer than $r$ are connected with a line and three points that are pairwise closer than $r$ form a triangle. In all figures, the initial data points are shown orange, edges connecting them are drawn as line segments, triangles are visualized as transparent blue fillings, and the values of $r$ are noted on top. (h) Barcode diagram showing the birth and death of components (red, solid) and holes (blue, dotted) as $r$ increases. Each bar spans an interval that begins at the $r$ value at which the respective component or hole is born and ends at the $r$ value at which it dies.

show visual representations of $\Xi_{r}$ for different values of $r$ in Fig. $3(\mathrm{a}-\mathrm{g})$.

As we vary $r$ from 0 to $\infty$, we keep track of the number of components and holes in $\Xi_{r}$. By a "component", we to refer to an individual point or a set of points and the edges that connect them and all triangles that fill the space in between. A "hole" is formed when a component is in the form of a loop with not-necessarily-distinct inner and outer boundaries. For example, in Fig. 3 (a) we have 14 components, whereas in Fig. 3 (b) we have 6 and in Fig. 3 (c) we have 1. In Fig. 3 (d), the single component of $\Xi_{r=5.07}$ forms a loop with a hole. As we further increase $r$, triangles begin to form (Fig. 3 (e-f)), and finally, the hole is completely filled with triangles at $r=13.30$ (Fig. $3(\mathrm{~g})$ ). This sequence of appearances, called "birth", and disappearances, called "death", of shapes can be encoded into diagrams such as the one in Fig. 3 (h). Fig. 3 (h) is called a "barcode diagram", where components and holes are represented by bars that span the interval of $r$ for which the respective object can be observed. Another graphical representation of the same information is the so-called "persistence diagram", on which the birth and death coordinates $\left(r_{B}, r_{D}\right)$ of components and holes are marked as shown in Fig. 4. We would like to note here that when two points are connected by an edge, which of the two points dies is ambiguous. This ambiguity, however, does not affect the barcode and persistence diagrams since both points appear at $r=0$.

Given a data set $\Xi$, the object that is of interest to us is the associated persistence diagram $\operatorname{PD}(\Xi)$. In general, the elements that are further away from the diagonal of a persistence diagram are said to be the more significant features of the data set, since they live for a longer range of resolutions. ${ }^{28}$ However, depending on the problem and what the resolution $r$ represents, features of interest may appear as short-lived elements as well. ${ }^{29,30}$

An important property of persistence diagrams is their stability: If the samples in the data set $\Xi$ are slightly perturbed, then the associated persistence diagram changes only slightly. We illustrate this in Fig. 4 where we show the persistence diagram associated with a data set sampled from the periodic orbit $\overline{1}$ (Fig. 2 (b), dashed) of the Rössler system next to the persistence diagram of a data set sampled from a trajectory that shadows it (Fig. 2 (b), solid). A proof of the stability of persistence diagrams can be found in Ref. 31.

We quantify the similarity of two persistence diagrams by defining a distance between them. Let us first define the set of diagonal elements $\Delta=\left\{\left(r_{B}, r_{D}\right) \in\right.$ $\left.[0, \infty) \times[0, \infty) \mid r_{B}=r_{D}\right\}$. These correspond to the "trivial" persistence diagram elements that are born and die at the same $r$ value. We can also denote the components and holes on a persistence diagram by the multisets

$$
\begin{aligned}
\mathrm{PD}_{i}= & \left\{\left(r_{B}, r_{D}\right)_{i, 1},\left(r_{B}, r_{D}\right)_{i, 2}, \ldots\left(r_{B}, r_{D}\right)_{i, E_{i}}\right\} \\
& \cup \Delta \cup \Delta \cup \Delta \ldots, \quad i \in\{0,1\}
\end{aligned}
$$

where $i=0$ corresponds to the components, $i=1$ corresponds to the holes, and $E_{i}$ is the number of respective elements in a diagram. We included the trivial sets with infinite multiplicity into the persistence diagrams for a reason which will be apparent soon. We are now in position to define a metric between the persistence diagrams $\mathrm{PD}_{i}^{(n)}$ and $\mathrm{PD}_{i}^{(m)}$. Let $\phi: \mathrm{PD}_{i}^{(n)} \rightarrow \mathrm{PD}_{i}^{(m)}$ be a bijection 

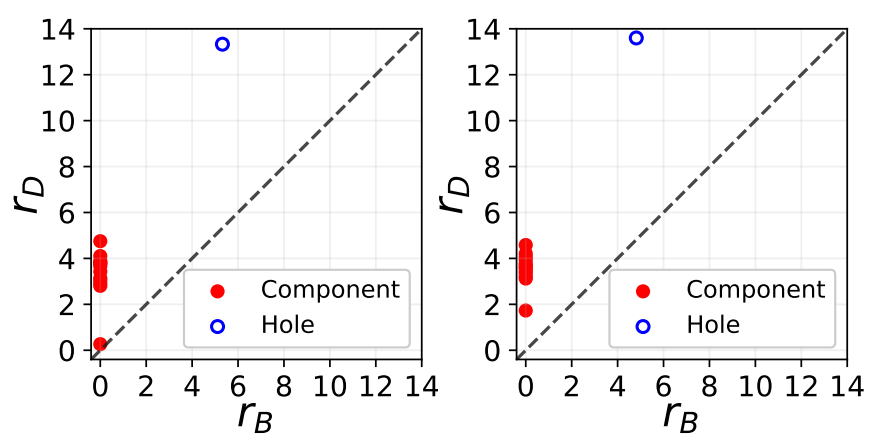

(a)

(b)

FIG. 4. (a) Persistence diagram corresponding to a data set sampled from the periodic orbit $\overline{1}$ of the Rössler system. (b) Persistence diagram obtained from a Rössler system trajectory which shadows the periodic orbit $\overline{1}$ as shown in Fig. 2 (b). The birth and death coordinates $\left(r_{B}, r_{D}\right)$ of the components and holes are marked red/solid and blue/hollow respectively.

that pairs each element of $\mathrm{PD}_{i}^{(n)}$ with exactly one element of $\mathrm{PD}_{i}^{(m)}$. We define the $p^{\text {th }}$ Wasserstein distance between $\mathrm{PD}_{i}^{(n)}$ and $\mathrm{PD}_{i}^{(m)}$ as

$$
W_{p}\left(\mathrm{PD}_{i}^{(n)}, \mathrm{PD}_{i}^{(m)}\right)=\inf _{\phi}\left[\sum_{\mu \in \mathrm{PD}_{i}^{(n)}}\|\mu-\phi(\mu)\|_{q}^{p}\right]^{1 / p}
$$

where $p \in[1, \infty], q \in[1, \infty]$, and \|\|$_{q}$ denotes the $L_{q^{-}}$ norm in $\mathbb{R}^{2}$. When $p=1$, the Wasserstein distance (14) can be understood as the smallest possible sum of the lengths of the line segments that can be drawn from the elements of $\mathrm{PD}_{i}^{(n)}$ to those of $\mathrm{PD}_{i}^{(m)}$. The addition of diagonal elements to the persistence diagrams makes it possible to compare different diagrams with possibly different number of nontrivial elements by allowing matching the nontrivial elements of one diagram to the diagonal of the other. Setting $p>1$ emphasizes the contributions from the elements that are further away from the diagonal in comparison to others, i.e. the ones that are more persistent against the changes in $r$.

This concludes our overview of the persistent homology concepts that we incorporate into state space persistence analysis. There are various algorithms and implementations for computing persistence diagrams and the Wasserstein distances (14) between them, which are not in the scope of the present work. For a review, we refer the interested reader to Ref. 27. In the applications that we present in section IV, we utilize the programs Ripser $^{32}$ for the computation of persistence diagrams and Hera ${ }^{33,34}$ for the computation of the Wasserstein distance.

\section{STATE SPACE PERSISTENCE ANALYSIS}

In this section, we list the basic steps of state space persistence analysis for capturing the symbolic dynamics of high-dimensional chaotic flows. Since the primary applications we have in mind are discretizations of nonlinear PDEs such as the Navier-Stokes equations, our presentation below is given for such systems.

\section{III.1. Symmetry reduction}

Nonlinear PDEs are usually equivariant under a certain set of symmetries such as translations, rotations, and reflections. These symmetries tend to obscure the dynamics by increasing the data volume since each solution has a set of symmetry copies that are also solutions. Furthermore, systems with continuous symmetries tend to have higher-dimensional invariant solutions such as relative periodic orbits, ${ }^{3,35}$ which are periodic orbits up to continuous symmetry transformations.

For state space persistence analysis, we assume that $\left(\mathcal{M}, f^{t}\right)$ is a symmetry-reduced realization of the dynamical system under consideration. In other words, before we begin our analysis, we carry out a symmetry-reducing coordinate transformation, which maps each symmetryequivalent solution of the system to a single representative $\xi \in \mathcal{M}$. This, in general, can be a nontrivial task. However, there has been considerable development in recent years following the introduction of the "first Fourier mode slice", ${ }^{36}$ which is a straightforward method for reducing the $S O(2)$ symmetry due to translation equivariance and periodic boundary conditions. Since its introduction, this method was adapted to the two-dimensional Kolmogorov flow, ${ }^{37}$ three-dimensional pipe flow, ${ }^{38,39}$ onedimensional Korteweg-de Vries equation, ${ }^{40}$ and pilotwave hydrodynamics. ${ }^{41}$ For a pedagogical introduction to the first Fourier mode slice, we refer the reader to Ref. 42. The reduction of discrete symmetries can also be nontrivial. The only discrete symmetry reduction method for high-dimensional systems in the literature known to us is the invariant polynomials for reflection-type symmetries. ${ }^{43}$ We present the symmetry reduction of the Kuramoto-Sivashinsky system in appendix A.

\section{III.2. Base set of periodic orbits}

We search for a base set of periodic orbits $\overline{\text { po }}=$ $\left\{\overline{\mathrm{po}}_{1}, \overline{\mathrm{po}}_{2}, \ldots, \overline{\mathrm{po}}_{M}\right\}$, with which we attempt to approximate chaotic dynamics. Generically, this set of periodic orbits can be found via recurrence-based searches ${ }^{4,5,18,19,44}$ or following bifurcations ${ }^{17,45}$ and unstable manifolds of known solutions. ${ }^{43,46}$ While there exist variational, ${ }^{46}$ Levenberg-Marquardt search-based, ${ }^{44}$ and possibly various other optimization methods for numerically locating unstable periodic orbits, the current 
community standard for very-high-dimensional flows is the Newton-Krylov-hookstep method of Viswanath. ${ }^{19}$

\section{III.3. Local persistence of periodic orbits}

We sample the states $\xi^{\left(\overline{\mathrm{po}}_{i}\right)}(t)$ on each periodic orbit $\overline{\mathrm{po}}_{i}$ with a constant sampling time $t_{s}$ and construct local projection bases $\left\{e_{1}^{\left(\overline{\mathrm{po}_{i}}\right)}, e_{2}^{\left(\overline{\mathrm{p}}_{i}\right)}, \ldots, e_{N_{i}}^{\left(\overline{\mathrm{po}}_{i}\right)}\right\}$ with the origins $O^{\left(\overline{\mathrm{po}}_{i}\right)}$ that locally capture the data points $\left\{\xi^{\left(\overline{\mathrm{p}}_{i}\right)}(0), \xi^{\left(\overline{\mathrm{po}}_{i}\right)}\left(t_{s}\right), \ldots, \xi^{\left(\overline{\mathrm{po}}_{i}\right)}\left(\left(N_{i}-1\right) t_{s}\right)\right\}$ of $\overline{\mathrm{po}}_{i}$. This can be achieved by a standard method such as principal component analysis (PCA). ${ }^{47}$ Note that with a fixed sampling time, each periodic orbit $\overline{\mathrm{po}}_{i}$ has a different number of samples $N_{i}$. Finally, we generate a catalog of persistence diagrams $\mathrm{PD}^{\left(\overline{\mathrm{po}}_{1}\right)}, \mathrm{PD}^{\left(\overline{\mathrm{po}}_{2}\right)}, \ldots, \mathrm{PD}^{\left(\overline{\mathrm{po}}_{M}\right)}$ from the local projections of the periodic orbit samples.

\section{III.4. Local persistence of chaotic trajectory segments}

Consider the data set

$\Xi^{(i)}(t)=\left\{\tilde{\xi}(t), \tilde{\xi}\left(t+t_{s}\right), \tilde{\xi}\left(t+2 t_{s}\right), \ldots, \tilde{\xi}\left(t+\left(N_{i}-1\right) t_{s}\right)\right\}$

with $N_{i}$ elements that are sampled from a chaotic trajectory starting at time $t$ and projected onto the local bases of the $i^{t h}$ periodic orbit. Let $\mathrm{PD}^{(i)}(t)$ be the persistence diagram obtained from this data set. We define the shadowing distance of a chaotic trajectory segment to the periodic orbit $\overline{\mathrm{po}}_{i}$ at time $t$ as the weighted sum

$$
\begin{aligned}
S^{(i)}(t) & =w_{0} W_{p}\left(\mathrm{PD}_{0}^{(i)}(t), \mathrm{PD}_{0}^{\left(\overline{\mathrm{po}}_{i}\right)}\right) \\
& +w_{1} W_{p}\left(\mathrm{PD}_{1}^{(i)}(t), \mathrm{PD}_{1}^{\left(\overline{\mathrm{po}}_{i}\right)}\right) .
\end{aligned}
$$

The adjustable weights $w_{0}$ and $w_{1}$ in (16) control the respective contributions of the components and the holes to the shadowing distance. In order to identify a chaotic trajectory's transient visits to the neighborhoods of the periodic orbits, we measure its shadowing distance from the base set of periodic orbits. The set of shadowing distances is the final output of state space persistence analysis. As we shall see in our applications, the shadowing distance of a chaotic trajectory segment to a periodic orbit becomes small when the trajectory segment has the same itinerary as the periodic orbit.

\section{NUMERICAL DEMONSTRATIONS}

In this section, we present two applications of state space persistence analysis. We begin with a controlled numerical experiment on the Rössler system.

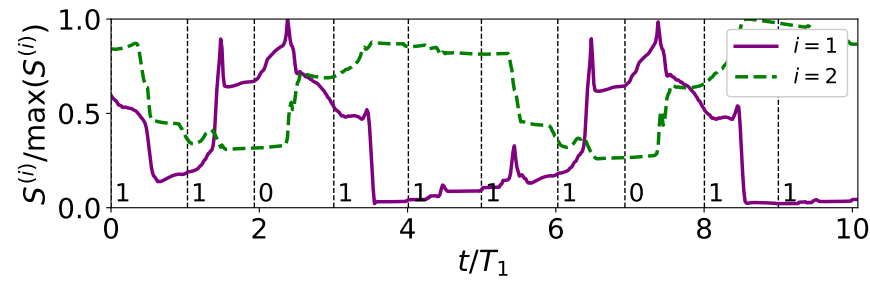

FIG. 5. Time series of the shadowing distances $S^{(1)}$ and $S^{(2)}$ between trajectory segments on the Rössler attractor and the periodic orbits $\overline{\mathrm{po}}_{1}=\overline{1}$ and $\overline{\mathrm{po}}_{2}=\overline{01}$. The time axis is in units of the period of the orbit $\overline{1}$. Symbol sequences printed at the bottom correspond to the windows marked by the vertical dashed lines and are read off from the Poincaré section.

\section{IV.1. Rössler system}

Since the Rössler system has no symmetries, we do not need a symmetry reduction, thus we begin our analysis by choosing $\left\{\overline{\mathrm{po}}_{1}=\overline{1}, \overline{\mathrm{po}}_{2}=\overline{01}\right\}$ (Fig. 2) as our base set. We also do not need local projection bases for these periodic orbits. Since the Rössler system is three-dimensional, we can carry out the calculation to follow in the full state space.

We sample a chaotic trajectory and the periodic orbits $\overline{1}$ and $\overline{01}$ with a constant sampling time of $t_{s}=0.1$ and compute the shadowing distances (16) of the chaotic trajectory from the periodic orbits using unit weights $w_{0}=w_{1}=1$ and the Wasserstein distance (14) with $p=q=2$. Fig. 5 shows the shadowing distances of a chaotic trajectory of the Rössler system from the periodic orbits $\overline{1}$ and $\overline{01}$. We normalized the shadowing distances in Fig. 5 by their respective maxima so that each time series takes values in the interval $[0,1]$. The symbols printed at the bottom, separated by vertical dashed line segments, form the itinerary of the chaotic trajectory and are read off from the Poincaré section. It is clear from Fig. 5 that the distance of the chaotic trajectory to the periodic orbit $\overline{1}$ has a dip when the chaotic trajectory's itinerary has a 1 . Similarly, the distance to the periodic orbit $\overline{01}$ has a dip when the itinerary has a symbol sequence 01 or 10 . These drops in the shadowing distance can be easily detected using a threshold and thus state space persistence analysis can indeed be used for inferring symbolic dynamics.

\section{IV.2. Kuramoto-Sivashinsky system}

The Kuramoto-Sivashinsky equation was originally proposed to model the phase dynamics of reactiondiffusion systems ${ }^{48}$ and instabilities of flame fronts. ${ }^{49}$ Owing to its computational simplicity, nowadays the $\mathrm{Ku}-$ ramoto-Sivashinsky system is frequently chosen as the testing ground for methods to study high-dimensional chaos and turbulence. ${ }^{43,44,50-53}$ In $(1+1)$ dimensions, 
the Kuramoto-Sivashinsky equation reads

$$
u_{t}=-u u_{x}-u_{x x}-u_{x x x x},
$$

where $x \in[-L / 2, L / 2)$ and $t \in[0, \infty)$ denote the space and time coordinates respectively and the subscripts imply partial derivatives. We interpret the scalar field $u(x, t)$ as the flame front velocity and assume the periodic boundary condition $u(x, t)=u(x+L, t)$. The domain length $L$ is the sole control parameter of the Kuramoto-Sivashinsky system, whose dynamics become chaotic when $L$ is large enough. ${ }^{43,44}$

The Kuramoto-Sivashinsky equation (17) is equivariant under continuous translations

$$
g_{x}(\delta x) u(x, t)=u(x-\delta x, t),
$$

where $\delta x \in[0, L)$, and the reflection

$$
\sigma u(x, t)=-u(-x, t) .
$$

As a consequence of the symmetries (18) and (19), the Kuramoto-Sivashinsky system has relative periodic orbits, which satisfy

$$
u_{p}=g f_{K S}^{T_{p}}\left(u_{p}\right),
$$

where $g \in\left\{g_{x}\left(\delta x_{p}\right), \sigma\right\}, \delta x_{p} \in[0, L)$, and $f_{K S}^{t}(u)$ is the flow map induced by the time evolution under (17). As we argued in section III.1, before the persistence analysis, we must obtain a symmetry-reduced representation for the Kuramoto-Sivashinsky system. This problem was addressed in Ref. 43, which combined the first Fourier mode slice method of Ref. 36 with an invariantpolynomial method to obtain a fully symmetry-reduced representation of the Kuramoto-Sivashinsky state space. Here, we follow a slightly different approach that does not introduce any new technique, therefore, we leave the details of this to appendix $\mathrm{A}$ and assume that we have a symmetry-reducing transformation $\hat{\xi}=\mathcal{R}(u)$ for all $u(x, t)$ of interest, such that

$$
\hat{\xi}=\mathcal{R}(u)=\mathcal{R}(g u),
$$

where $g \in\left\{g_{x}(\delta x), \sigma\right\}$ and $\delta x \in[0, L)$. Once we obtain the symmetry-reducing transformation (21), the symmetry-reduced flow is obtained straightforwardly as

$$
\hat{\xi}(t)=\hat{f}^{t}(\hat{\xi}(0))=\mathcal{R}\left(f_{K S}^{t}\left(\mathcal{R}^{-1}(\hat{\xi}(0))\right)\right) .
$$

Note that the inverse transformation $u=\mathcal{R}^{-1}(\hat{\xi})$ cannot be unique, since the symmetry reduction (21) maps all symmetry-equivalent states to one. However, this nonuniqueness makes no difference in the symmetry-reduced flow (22), thus, any one of the available symmetry-equivalent inverses can be taken.

After the symmetry reduction (21), by definition, the relative periodic orbits (20) become periodic orbits (5). By numerically following the unstable manifolds of relative periodic orbits, Ref. 43 presented evidence that the chaotic dynamics of the Kuramoto-Sivashinsky system at $L=21.7$ take place in the vicinity of four relative periodic solutions all of which are unstable. We show these orbits along with a chaotic trajectory in Fig. 6 as a PCA projection. The projection bases were obtained as the first three principal components corresponding to a chaotic trajectory of temporal length $t_{f}=10^{5}$, sampled at the sampling time $t_{s}=10$. Four periodic orbits

$$
\overline{\mathrm{po}}=\left\{\overline{\mathrm{po}}_{1}, \overline{\mathrm{po}}_{2}, \overline{\mathrm{po}}_{3}, \overline{\mathrm{po}}_{4}\right\}
$$

with periods $T_{1}=10.11, T_{2}=32.37, T_{3}=36.70, T_{4}=$ 36.08 , which we show in Fig. 6 , form the base set for the state space persistence analysis of the Kuramoto-Sivashinsky system. In order to confirm that our chaotic data set is long enough to cover the attractor of the system sufficiently, we reproduced Fig. 6 with random initial conditions and found the resulting projections to be practically indistinguishable. We generated these initial conditions by populating the Fourier coefficients of $u(x, 0)$ with random numbers drawn from the standard normal distribution.

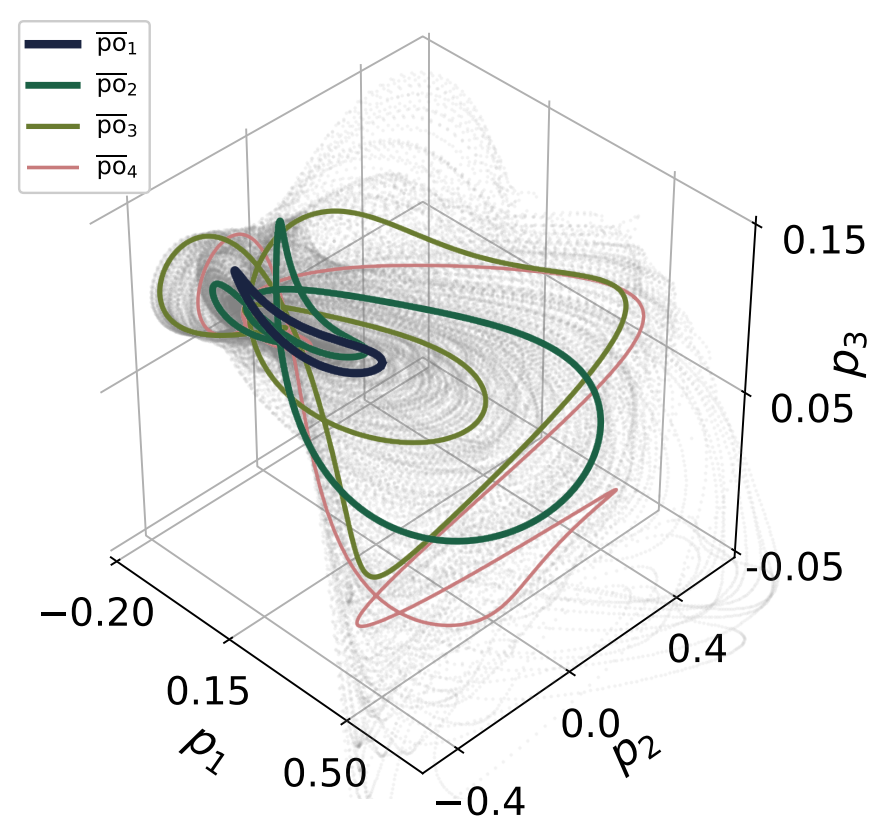

FIG. 6. A chaotic trajectory (gray dots) and four periodic orbits (different colors/thickness) of the Kuramoto-Sivashinsky system projected onto three leading PCA directions obtained from a chaotic data set.

We sample each orbit in our base set (23) with the constant sampling time $t_{s}=0.5$ and use these samples to generate local PCA bases and persistence diagrams for each periodic orbit as described in section III.3. In Fig. 7 (a), we show the shadowing distances (16) of a chaotic trajectory from the four periodic orbits of the Kuramoto-Sivashinsky system as a function of time. Similar to Fig. 5, we normalized each shadowing distance by its maximum. In computing these distances, we used unit 
weights $w_{0}=w_{1}=1$ in (16) and the Wasserstein distance (14) with $p=q=2$.

When the shadowing distance (16) to a particular periodic orbit is small, we expect to find the chaotic trajectory segment to have a shape similar to that of the respective periodic orbit. We illustrate this on the local projections of Fig. 7 (c-f), where we show chaotic trajectory segments of different durations with initial conditions corresponding to the local minima of the shadowing distances in Fig. 7 (b) along with the projections of the periodic orbits. As further evidence, we show the spacetime visualizations of the shadowing trajectory segments next to the periodic orbits in Fig. $7(g-j)$, where the amplitude of the scalar field $u(x, t)$ is color-coded. The time interval shown in Fig. 7 (g) spans approximately five periods of $\overline{\mathrm{po}}_{1}$, whereas for the rest of the periodic orbits one period is shown in Fig. $7(\mathrm{~h}-\mathrm{j})$. Note that since the space-time visualizations are not symmetry reduced, the relative periodic orbits arrive at a symmetry-transformed state after one period. This can easily be seen on Fig. 7 (j), where the final state is the initial state, shifted in space by $\delta x \approx 12.07$. In Fig. 7 (g-i), the initial and final states are related by reflection.

\section{DISCUSSION}

We presented the results of state space persistence analysis in the Rössler and the Kuramoto-Sivashinsky systems where in both cases, we saw that the shadowing distance successfully captured the shape similarities between periodic orbits and chaotic trajectory segments. We chose the Rössler system for the first application of state space persistence analysis in order to avoid technical difficulties associated with PDEs and illustrate the core concepts of our method on a simple example. With this in mind, we considered only two periodic orbits, namely $\overline{1}$ and $\overline{01}$, in our analysis. We would like to note the limitations that come with this choice: In general, the trajectories of the Rössler system can have itineraries containing the symbol sequence 00 (see Fig. 2 (a)), however, none of the periodic orbits $\overline{1}$ or $\overline{01}$ contain this symbol sequence. Thus, the base set of periodic orbits that we considered in section IV.1 cannot capture trajectory segments which are associated with the symbol sequence 00 . We could have captured some these motions by including the periodic orbit $\overline{001}$ into our analysis, however, we refrained from doing so for the clarity of the presentation.

The success of state space persistence analysis in the Kuramoto-Sivashinsky system reveals the true potential of the method for the study of high-dimensional systems. It is important to note that in Fig. 7 (a) at almost all times, at least one of the shadowing distances is less than 0.5 and has a local minimum. This demonstrates that the spatiotemporally chaotic Kuramoto-Sivashinsky dynamics can be approximated by a Markov chain based on the four periodic solutions. Notice also that some of the step-like minima of the shadowing distances in Fig. 7 (a) coincide: For example, both $S^{(1)}$ and $S^{(2)}$ start at a low value with similar instances in the future. This suggests that $\overline{\mathrm{po}}_{1}$ and $\overline{\mathrm{po}}_{2}$ could be related through a bifurcation. Indeed, $\overline{\mathrm{po}}_{2}$ appears on the unstable manifold of $\overline{\mathrm{po}}_{1}$ at a lower value of the control parameter $L$ as demonstrated in Ref. 43. Another important observation to make on Fig. 7 (a) is that all dips in $S^{(4)}$ are preceded by those of $S^{(3)}$. This suggests that $\overline{\mathrm{po}}_{3}$ admits a symmetry-breaking instability since $\overline{\mathrm{po}}_{4}$ has a nonzero spatial drift, see Fig. 7 (j). A detailed investigation and periodic-orbit-based modeling of the Kuramoto-Sivashinsky dynamics will be a subject of a future study.

In order to test our method's robustness against the choice of norm, we partially repeated our calculations using "randomly modified" norms. To this end, we defined

$$
\left\langle\xi^{(i)}, \xi^{(j)}\right\rangle_{R^{(l)}}=\sum_{k=1}^{D} R_{k}^{(l)} \xi_{k}^{(i)} \xi_{k}^{(j)},
$$

where $R_{k}^{(l)}$ are positive pseudorandom numbers, the sum of which is equal to $D$, the system dimension. Note that if we choose $R_{k}=1$, we recover the $L_{2}$-norm (4). We found that the shadowing distances obtained with modified norms looked qualitatively similar to those in Fig. 5 and Fig. 7. Although it is beyond the scope of the present work, we speculate that the robustness of the shadowing distance (16) against the modifications (24) of the inner product could potentially be established rigorously, with techniques similar to those used to prove the stability of persistence diagrams. ${ }^{31}$

In our analyses, we chose the sampling time $t_{s}$, Wasserstein distance degree $p$, and the shadowing distance weights $w_{i}$ through numerical experimentation. We first produced the data for these experiments by generating shadowing trajectories from the initial conditions corresponding to slight perturbations to periodic orbits as in Fig. 2 (b). We then generated persistence diagrams and computed the associated shadowing distances for different choices of parameters and then settled with the ones that yielded the expected shadowing signals. We chose the sampling time $t_{s}$ via a trade-off: When $t_{s}$ was too long the persistence diagrams missed significant topological features of underlying trajectories; whereas when the sampling time was too short the persistence diagrams associated with the periodic orbits and shadowing trajectories differed significantly. In choosing the sampling time, we avoided both of these extremes. Apart from the Wasserstein distance degree $p=2$ that we used, we also tried $p=1$, which resulted in shadowing signals with smoother variations in time. Consequently, we decided to use $p=2$, since sharper variations in time would be more amenable to shadowing detection with a thresholding algorithm. Besides the unit weights $w_{0}=w_{1}=1$ in the shadowing distance (16), we also tried $w_{0,1}=\left[W_{p}\left(\mathrm{PD}_{0,1}^{0}, \mathrm{PD}_{0,1}^{\overline{\mathrm{po}}_{i}}\right)\right]^{-1}$, where $\mathrm{PD}_{0,1}^{0}$ are trivial persistence diagrams with diagonal elements only. This 


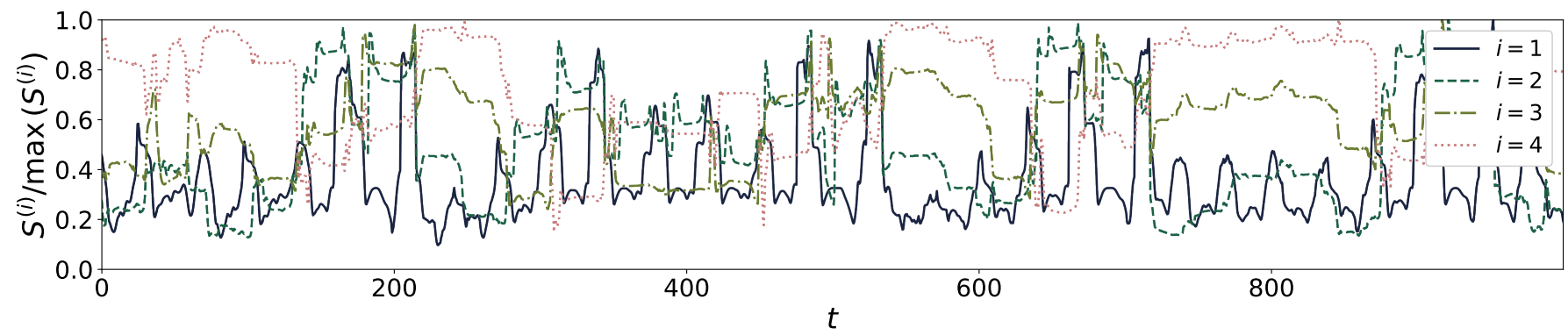

(a)

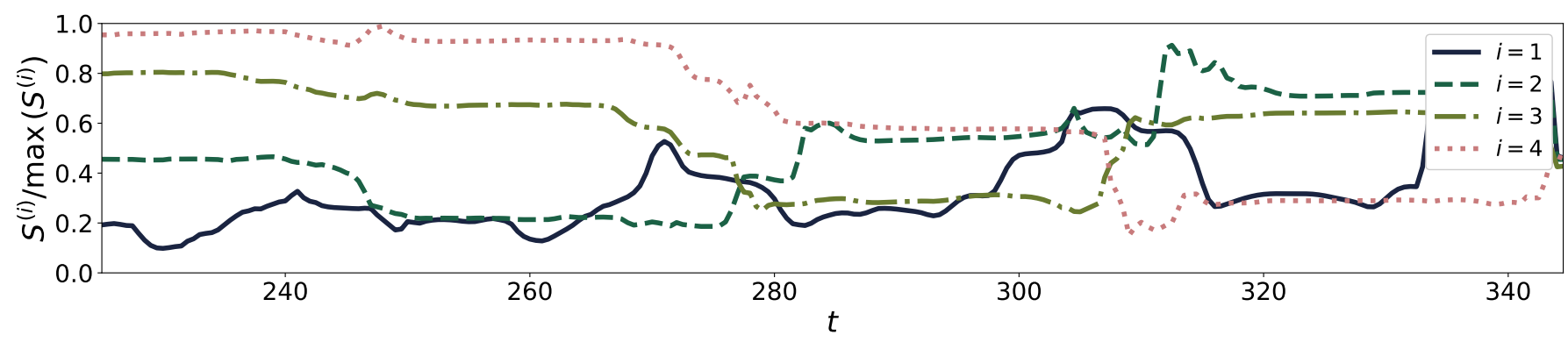

(b)

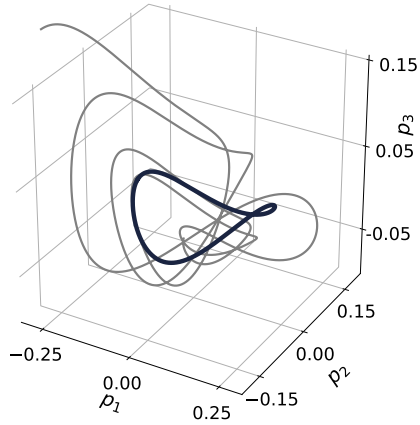

(c)

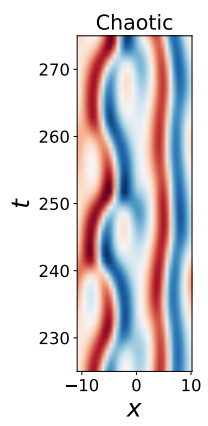

(g)

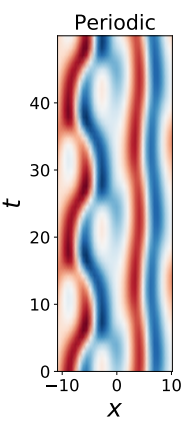

(h)

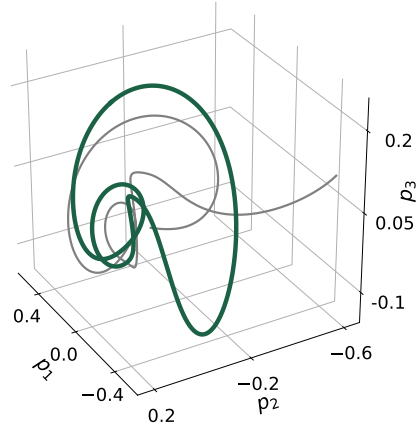

(d)
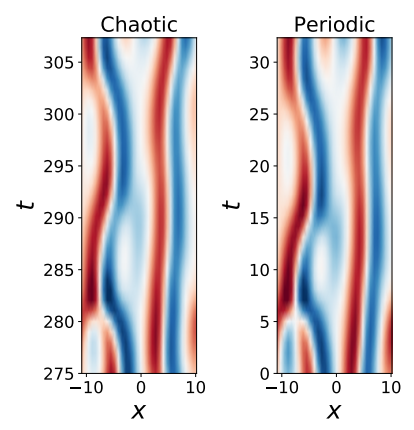

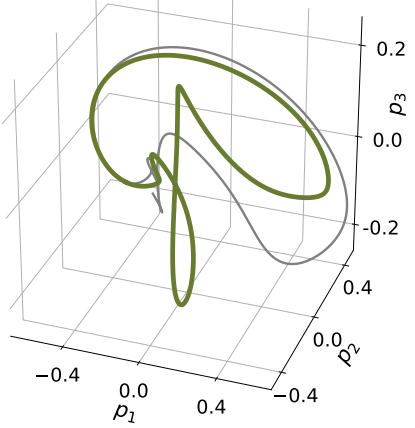

(e)

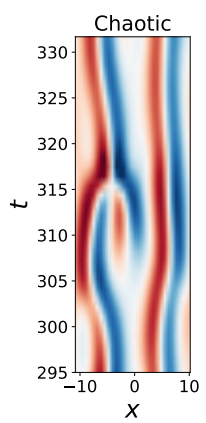

(i)

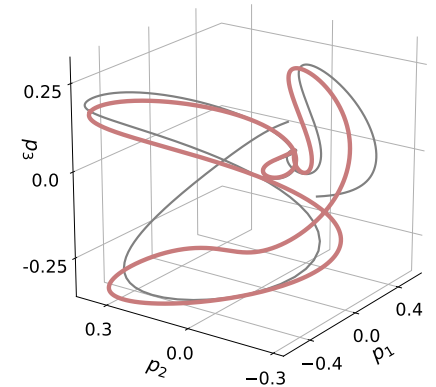

(f)
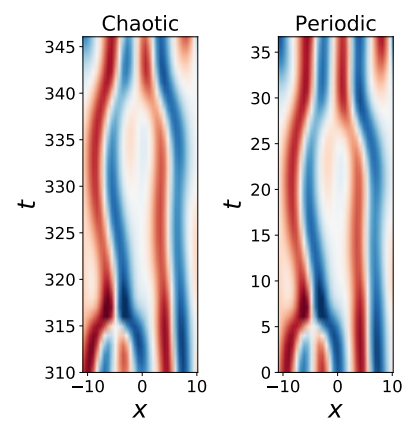

(j)

FIG. 7. (a) Shadowing distances $S^{(i)}$ of a chaotic trajectory to the periodic orbits $\overline{\mathrm{po}}_{i}, i \in\{1,2,3,4\}$ of the KuramotoSivashinsky system. (b) Zoom into the time interval of (a) that corresponds to the visualizations in (c-j). (c-f) Examples of shadowing trajectory segments which correspond to the time interval $t \in[225,345]$, shown in (b), visualized as local projections of chaotic trajectories segments (gray) onto the local PCA bases of the respective periodic orbits $\overline{\mathrm{po}}_{1}, \ldots, \overline{\mathrm{po}}_{4}(\mathrm{colors} / \mathrm{bold})$ that are shadowed. $(\mathrm{g}-\mathrm{j})$ Chaotic trajectory segments next to the periodic solutions $\overline{\mathrm{po}}_{1}, \ldots, \overline{\mathrm{po}}_{4}$ that they shadow, visualized in space-time by color-coding the amplitude of $u(x, t)$. Time intervals of the shadowing trajectory segments (g-j) in space-time visualizations are the same intervals shown in the local projections $(\mathrm{c}-\mathrm{f})$. 
choice overemphasized holes, which resulted in the corresponding shadowing distance time series missing some of the symbol assignments in the Rössler system.

\section{CONCLUSION AND PERSPECTIVES}

In this paper, we introduced state space persistence analysis for inferring the symbolic dynamics of a chaotic time series by quantifying the shape similarity of chaotic trajectory segments and periodic solutions of the system. Our starting motivation was to have a tool for understanding high-dimensional chaos in terms of the periodic solutions of a system and we demonstrated that state space persistence analysis can be utilized for this purpose by successfully applying it to the spatiotemporally chaotic Kuramoto-Sivashinsky system. We are now in position to apply our method to problems that are computationally much more challenging, such as the simulations of the Navier-Stokes equations in three dimensions.

We would like to mention that our use of persistence is similar in spirit to the "Sliding Windows and 1dimensional Persistence Scoring" (SW1PerS) method, ${ }^{54}$ in which one constructs delay embeddings of time series data before carrying the persistence computation in order to detect periodicities in the data. In our case, we do not need a delay embedding since we assume that we have access to the complete state space information. Moreover, instead of trying to detect periodicities in the signal, we try to identify similarities to a certain precomputed set of periodic solutions in state space persistence analysis. One can imagine applications in which the two methods are mixed. For example, if one is searching for shadowing in a laboratory experiment in which the complete state measurement is not available, state space persistence analysis could be carried out on a delay embedding. Another interesting hybrid application could be searching for periodic solutions using the state space persistence of time series data.

In this paper, we opted for the simplicity of the presentation rather than fine-tuning our tools. As a consequence, there are a lot of aspects of state space persistence analysis that could potentially be optimized for different settings. As we discussed in section V, the parameters such as sampling time, Wasserstein distance degree, and shadowing distance weights should be chosen according to the specific properties of a problem. Our exploration of this free parameter space was by no means exhaustive and we expect that these choices will need to be revisited when applying state space persistence analysis in different settings. Establishing the guidelines for this purpose will be a topic of our future research.

\section{ACKNOWLEDGMENTS}

We are grateful to Predrag Cvitanović for his comments on an early version of this manuscript and to the anonymous referee, whose suggestions helped us to improve this paper.

\section{Appendix A: State space of the Kuramoto-Sivashinsky system}

We begin our numerical formulation by plugging the Fourier expansion $u=\sum_{k} \tilde{u}_{k}(t) e^{i q_{k} x}$, where $q_{k}=2 \pi k / L$ and $k=\ldots,-2,-1,0,1,2, \ldots$, into the Kuramoto-Sivashinsky equation (17) in order to obtain the infinite set of ODEs

$$
\dot{\tilde{u}}_{k}=\left(q_{k}^{2}-q_{k}^{4}\right) \tilde{u}_{k}-i \frac{q_{k}}{2} \sum_{m=-\infty}^{+\infty} \tilde{u}_{m} \tilde{u}_{k-m} .
$$

Noting that the $0^{\text {th }}$ Fourier mode $\tilde{u}_{0}$ is decoupled from the rest and $\tilde{u}_{-k}=\tilde{u}_{k}^{*}$ due to the realness of $u(x, t)$, a truncated state vector of the Kuramoto-Sivashinsky system can be expressed as

$$
\xi=\left(a_{1}, b_{1}, a_{2}, b_{2}, \ldots, a_{N}, b_{N}\right),
$$

where $\left(a_{k}, b_{k}\right)=\left(\operatorname{Re} \tilde{u}_{k}, \operatorname{Im} \tilde{u}_{k}\right)$ and $N$ is the highest Fourier mode that is kept in the expansion. In our computations, we used $N=15$, the adequacy of which was demonstrated in Ref. 44. In our codes, the nonlinear term in (A1) is computed pseudospectrally ${ }^{55}$ and the time-stepping is carried out using the general-purpose integrator odeint from scipy, ${ }^{56}$ which itself is a wrapper of 1soda from the ODEPACK library. ${ }^{57}$

It is straightforward to confirm that the action of the symmetries (18) and (19) on the real-valued state space coordinates (A2) are

$$
g_{x}(\delta x)\left(a_{k}, b_{k}\right)=R(-k \phi)\left(a_{k}, b_{k}\right)
$$

and

$$
\sigma\left(a_{k}, b_{k}\right)=\left(-a_{k}, b_{k}\right),
$$

where $\phi=2 \pi \delta x / L$ and $R(\theta)$ is the $2 \times 2$ rotation matrix

$$
R(\theta)=\left(\begin{array}{cc}
\cos \theta & -\sin \theta \\
\sin \theta & \cos \theta
\end{array}\right) .
$$

The first Fourier mode slice method of Ref. 36 fixes the polar angle on the subspace spanned by the first Fourier mode, i.e. $\left(a_{1}, b_{1}\right)$, in order to eliminate the spatial drifts. It was already demonstrated in Ref. 36 that such a transformation in the Kuramoto-Sivashinsky system leads to rapid fluctuations in time, which, in general, could be regularized by rescaling the time variable. Here, we tackle this problem by a different approach, which we found simpler to use in state space persistence analysis. Let $\xi$ be a generic state of the Kuramoto-Sivashinsky system with nonzero components in the second Fourier mode subspace, i.e. $a_{2}^{2}+b_{2}^{2}>0$. We search for a shifted state

$$
\gamma=g_{x}(-\delta \hat{x}) \xi
$$


such that $\gamma=\left(\hat{a}_{1}^{\prime}, \hat{b}_{1}^{\prime}, \hat{a}_{2}^{\prime}, \hat{b}_{2}^{\prime}, \ldots\right)$ has

$$
\hat{a}_{2}^{\prime}=0, \hat{b}_{2}^{\prime}>0 \text {. }
$$

Transforming to $\gamma(\mathrm{A} 6)$ eliminates the continuous translation degree of freedom by fixing the phase of the second Fourier mode. However, it does not fully reduce this symmetry since if $\gamma(\mathrm{A} 6)$ satisfies (A7) so does

$$
g_{x}(L / 2) \gamma=\left(-\hat{a}_{1}^{\prime},-\hat{b}_{1}^{\prime}, 0, \hat{b}_{2}^{\prime},-\hat{a}_{3}^{\prime},-\hat{b}_{3}^{\prime}, \hat{a}_{4}^{\prime}, \hat{b}_{4}^{\prime}, \ldots\right) .
$$

In other words, transformation to (A6) turns the continuous translation symmetry into a discrete one. As we shall see, this discrete symmetry can be reduced by the construction of invariant polynomials similar to those introduced in Ref. 43.

After the transformation (A6), the state space has two discrete symmetries, whose actions flip the signs of a subset of the state space coordinates. Notice that the action of the reflection $\sigma$ (A4) does not break the condition (A7) since $\hat{\xi}^{\prime}$ has $a_{2}^{\prime}=0$ and $b_{2}$ is invariant under $\sigma$. Following the recipe of Ref. 43 , we can define a reflection-reduced state vector as

$$
\begin{aligned}
\rho= & \left(a_{1}^{\prime 2}-a_{3}^{\prime 2}, b_{1}^{\prime}, b_{2}^{\prime}, a_{1}^{\prime} a_{3}^{\prime}, b_{3}^{\prime}, a_{3}^{\prime} a_{4}^{\prime}, b_{4}^{\prime}, a_{4}^{\prime} a_{5}^{\prime}, b_{5}^{\prime}\right. \\
& \left.a_{5}^{\prime} a_{6}^{\prime}, b_{6}^{\prime}, a_{6}^{\prime} a_{7}^{\prime}, b_{7}^{\prime}, a_{7}^{\prime} a_{8}^{\prime}, b_{8}^{\prime} \ldots\right),
\end{aligned}
$$

where we omitted $a_{2}^{\prime}$, since it is set to 0 . Note that (A9) is invariant under the sign change of all $a_{k}$ and not invariant under the sign change of any other subset of $a_{k} \mathrm{~s}$.

We can now turn our attention to the discrete symmetry due to the half-domain shift (A8). We should first find the representation of this symmetry on the reflectioninvariant polynomial coordinates (A9). Denoting the $k^{\text {th }}$ elements of (A9) by $\rho_{k}$ it follows from inspection that

$$
\begin{aligned}
g_{x}(L / 2) \rho= & \left(\rho_{1},-\rho_{2}, \rho_{3}, \rho_{4},-\rho_{5},-\rho_{6}, \rho_{7},-\rho_{8},-\rho_{9},\right. \\
& \left.-\rho_{10}, \rho_{11},-\rho_{12},-\rho_{13},-\rho_{14}, \rho_{15} \ldots\right) .(\text { A } 10)
\end{aligned}
$$

Beginning with $\rho_{8}$, every element of $\rho$ except $\left\{\rho_{11}, \rho_{15}, \rho_{19}, \rho_{23}, \rho_{27}, \ldots\right\} \quad$ (every fourth element) changes its sign under the action of $g_{x}(L / 2)$. Thus, we can write the final invariant polynomial coordinates as

$$
\begin{aligned}
\hat{\xi}= & \left(\rho_{1}, \rho_{2}^{2}-\rho_{5}^{2}, \rho_{3}, \rho_{4}, \rho_{2} \rho_{5}, \rho_{5} \rho_{6}, \rho_{7}, \rho_{6} \rho_{8}, \rho_{8} \rho_{9}, \rho_{9} \rho_{10},\right. \\
& \left.\rho_{11}, \rho_{10} \rho_{12}, \rho_{12} \rho_{13}, \rho_{13} \rho_{14}, \rho_{15}, \ldots\right) .
\end{aligned}
$$

While it might appear complicated, the invariant polynomial coordinates (A9) and (A11) follow a regular pattern, and thus, are straightforward to implement. We used the symmetry-invariant state space coordinates (A11) to obtain the results of section IV.2.

\footnotetext{
${ }^{1}$ R. L. Devaney. A First Course In Chaotic Dynamical Systems: Theory And Experiment. Studies in Nonlinearity. Avalon Publishing, 1992.

${ }^{2}$ K. T. Alligood, T. D. Sauer, and J. A. Yorke. Chaos: An Introduction to Dynamical Systems. Springer Berlin Heidelberg, Berlin, Heidelberg, 1997.

${ }^{3}$ P. Cvitanović, R. Artuso, R. Mainieri, G. Tanner, and G. Vattay. Chaos: Classical and Quantum. Niels Bohr Inst., Copenhagen, 2019. ChaosBook.org.
}

${ }^{4}$ P. Cvitanović and J. F. Gibson. Geometry of turbulence in wallbounded shear flows: Periodic orbits. Phys. Scr., T142:014007, 2010.

${ }^{5}$ N. B. Budanur, K. Y. Short, M. Farazmand, A. P. Willis, and P. Cvitanović. Relative periodic orbits form the backbone of turbulent pipe flow. J. Fluid Mech., 833:274-301, 2017. arXiv:1705.03720.

${ }^{6}$ S. H. Strogatz. Nonlinear Dynamics and Chaos. Perseus Books, Cambridge, MA, 2000.

${ }^{7}$ S. Smale. Diffeomorphisms with many periodic points. In Differential and combinatorial topology: A symposium in honor of Marston Morse, volume 27, pages 63-80. Princeton University Press, 1965.

${ }^{8}$ S. Smale. Differentiable dynamical systems. Bull. Amer. Math. Soc., 73:747-817, 1967.

${ }^{9}$ G.D. Birkhoff. Nouvelles recherches sur les systèmes dynamiques. Mémoriae Pont. Acad. Sci. Novi Lyncaei, 1:85-216, 1935.

${ }^{10}$ E. N. Lorenz. Deterministic nonperiodic flow. J. Atmos. Sci., 20:130-141, 1963

${ }^{11}$ O. E. Rössler. An equation for continuous chaos. Phys. Lett. A, $57: 397,1976$.

${ }^{12} \mathrm{D}$. Viswanath. Symbolic dynamics and periodic orbits of the Lorenz attractor. Nonlinearity, 16:1035-1056, 2003.

${ }^{13}$ C. Letellier, P. Dutertre, and B. Maheu. Unstable periodic orbits and templates of the rössler system: Toward a systematic topological characterization. Chaos, 5(1):271-282, 1995.

${ }^{14} \mathrm{P}$. Cvitanović. Recurrent flows: the clockwork behind turbulence. J. Fluid Mech. Focus Fluids, 726:1-4, 2013.

${ }^{15}$ E. M. Cherry, F. H. Fenton, T. Krogh-Madsen, S. Luther, and U. Parlitz. Introduction to focus issue: Complex cardiac dynamics. Chaos, 27(9):093701, 2017.

${ }^{16} \mathrm{M}$. Doebeli and I. Ispolatov. Chaos and unpredictability in evolution. Evolution, 68(5):1365-1373, 2014. arXiv:1309.6261.

${ }^{17}$ T. Kreilos and B. Eckhardt. Periodic orbits near onset of chaos in plane Couette flow. Chaos, 22:047505, 2012. arXiv:1205.0347.

${ }^{18}$ D. Lucas and R. R. Kerswell. Recurrent flow analysis in spatiotemporally chaotic 2-dimensional Kolmogorov flow. Phys. Fluids, 27:518-554, 2015. arXiv:1406.1820.

${ }^{19}$ D. Viswanath. Recurrent motions within plane Couette turbulence. J. Fluid Mech., 580:339-358, 2007. arXiv:physics/0604062.

${ }^{20}$ C. Epstein, G. Carlsson, and H. Edelsbrunner. Topological data analysis. Inverse Probl., 27(12):120201, 2011.

${ }^{21} \mathrm{H}$. Edelsbrunner and J. Harer. Persistent homology-a survey, pages 257-282. Am. Math. Soc., Providence, 2008.

${ }^{22}$ C. M. Topaz, L. Ziegelmeier, and T. Halverson. Topological data analysis of biological aggregation models. PLOS ONE, 10(5):126, 05 2015. arXiv:1412.6430.

${ }^{23}$ M. Kramár, R. Levanger, J. Tithof, B. Suri, M. Xu, M. Paul, M. F. Schatz, and K. Mischaikow. Analysis of kolmogorov flow and rayleigh-bénard convection using persistent homology. Physica D, 334:82-98, 2016. arXiv:1505.06168.

${ }^{24}$ J. Garland, E. Bradley, and J. D. Meiss. Exploring the topology of dynamical reconstructions. Physica D, 334:49-59, 2016. arXiv:1506.01128.

${ }^{25}$ A. Myers, E. Munch, and F. A. Khasawneh. Persistent homology of complex networks for dynamic state detection. Phys. Rev. E, 100:022314, Aug 2019. arXiv:1904.07403.

${ }^{26} \mathrm{H}$. Edelsbrunner and J. Harer. Computational topology: an introduction. American Mathematical Soc., 2010.

${ }^{27}$ N. Otter, M. A. Porter, U. Tillmann, P. Grindrod, and H. A. Harrington. A roadmap for the computation of persistent homology. EPJ Data Sci, 6(1):17, 2017. arXiv:1506.08903.

${ }^{28}$ G. Carlsson. Topology and data. Bull. Amer. Math. Soc., 46(2):255-308, 2009.

${ }^{29}$ M. Feng and M. A. Porter. Persistent homology of geospatial data: A case study with voting. preprint, 2019. arXiv:1902.05911.

${ }^{30}$ B. J. Stolz, H. A. Harrington, and M. A. Porter. Persistent homology of time-dependent functional networks constructed from coupled time series. Chaos: An Interdisciplinary Journal of Nonlinear Science, 27(4):047410, 2017. arXiv:1605.00562. 
${ }^{31}$ D. Cohen-Steiner, H. Edelsbrunner, and J. Harer. Stability of persistence diagrams. Discrete Comput Geom., 37(1):103-120, 2007.

${ }^{32}$ U. Bauer. Ripser, 2016. https://github.com/Ripser/ripser.

${ }^{33}$ M. Kerber, D. Morozov, and A. Nigmetov. Hera, 2016. https: //bitbucket.org/grey_narn/hera.

${ }^{34}$ M. Kerber, D. Morozov, and A. Nigmetov. Geometry helps to compare persistence diagrams. In Proceedings of the Eighteenth Workshop on Algorithm Engineering and Experiments (ALENEX), pages 103-112, 2016. arXiv:1606.03357.

${ }^{35} \mathrm{P}$. Chossat and R. Lauterbach. Methods in Equivariant Bifurcations and Dynamical Systems. World Scientific, Singapore, 2000.

${ }^{36}$ N. B. Budanur, P. Cvitanović, R. L. Davidchack, and E. Siminos. Reduction of the $\mathrm{SO}(2)$ symmetry for spatially extended dynamical systems. Phys. Rev. Lett., 114:084102, 2015. arXiv:1405.1096.

${ }^{37} \mathrm{M}$. Farazmand. An adjoint-based approach for finding invariant solutions of Navier-Stokes equations. J. Fluid Mech., 795:278312, 2016. arXiv:1508.06363.

${ }^{38}$ N. B. Budanur and B. Hof. Heteroclinic path to spatially localized chaos in pipe flow. J. Fluid Mech., 827, R1, 2017. arXiv:1703.10484

${ }^{39}$ N. B. Budanur and B. Hof. Complexity of the laminar-turbulent boundary in pipe flow. Phys. Rev. Fluids, 3:054401, 2018. arXiv:1802.01918.

${ }^{40} \mathrm{~S}$. Mowlavi and T. Sapsis. Model order reduction for stochastic dynamical systems with continuous symmetries. SIAM J. Sci. Comput, 40(3):A1669-A1695, 2018. arXiv:1704.06352.

${ }^{41}$ N. B. Budanur and M. Fleury. State space geometry of the chaotic pilot-wave hydrodynamics. Chaos, 29(1):013122, 2019. arXiv: 1812.09011.

${ }^{42}$ N. B. Budanur, D. Borrero-Echeverry, and P. Cvitanović. Periodic orbit analysis of a system with continuous symmetry - a tutorial. Chaos, 25:073112, 2015. arXiv:1411.3303.

${ }^{43}$ N. B. Budanur and P. Cvitanović. Unstable manifolds of relative periodic orbits in the symmetry-reduced state space of the Kuramoto-Sivashinsky system. J. Stat. Phys., 167(3):636-655, 2017. arXiv:1509.08133.

${ }^{44}$ P. Cvitanović, R. L. Davidchack, and E. Siminos. On the state space geometry of the Kuramoto-Sivashinsky flow in a periodic domain. SIAM J. Appl. Dyn. Syst., 9:1-33, 2009. arXiv:0709.2944.
${ }^{45}$ F. Christiansen, P. Cvitanović, and V. Putkaradze. Spatiotemporal chaos in terms of unstable recurrent patterns. Nonlinearity, 10:55-70, 1997. arXiv:chao-dyn/9606016.

${ }^{46} \mathrm{Y}$. Lan and P. Cvitanović. Unstable recurrent patterns in Kuramoto-Sivashinsky dynamics. Phys. Rev. E, 78:026208, 2008. arXiv:0804.2474.

${ }^{47}$ I. T. Jolliffe. Principal Component Analysis, Second Edition. Springer, 2002.

${ }^{48} \mathrm{Y}$. Kuramoto and T. Tsuzuki. Persistent propagation of concentration waves in dissipative media far from thermal equilibrium. Progr. Theor. Phys., 55:365, 1976.

${ }^{49}$ G. I. Sivashinsky. Nonlinear analysis of hydrodynamical instability in laminar flames - I. Derivation of basic equations. Acta Astronaut., 4:1177, 1977.

${ }^{50}$ P. Holmes, J. L. Lumley, and G. Berkooz. Turbulence, Coherent Structures, Dynamical Systems and Symmetry. Cambridge Univ. Press, Cambridge, 1996.

${ }^{51}$ D. Goluskin and G. Fantuzzi. Bounds on mean energy in the Kuramoto-Sivashinsky equation computed using semidefinite programming. Nonlinearity, 32(5):1705-1730, 2019. arXiv: 1802.08240.

52 J. Pathak, A. Wikner, R. Fussell, S. Chandra, B. R. Hunt, M. Girvan, and E. Ott. Hybrid forecasting of chaotic processes: Using machine learning in conjunction with a knowledge-based model. Chaos, 28(4):041101, 2018. arXiv:1803.04779.

${ }^{53}$ J. Pathak, Z. Lu, B. R. Hunt, M. Girvan, and E. Ott. Using machine learning to replicate chaotic attractors and calculate lyapunov exponents from data. Chaos, 27(12):121102, 2017. arXiv:1710.07313.

${ }^{54}$ J. A. Perea and J. Harer. Sliding windows and persistence: An application of topological methods to signal analysis. Found. Comput. Math., 15(3):799-838, 2015. arXiv:1307.6188.

${ }^{55}$ C. Canuto, M. Y. Hussaini, A. Quarteroni, and T. A. Zang. Spectral Methods: Evolution to Complex Geometries and Applications to Fluid Dynamics. Springer, New York, 2007.

${ }^{56}$ E. Jones, T. Oliphant, P. Peterson, et al. SciPy: Open source scientific tools for Python, 2001.

${ }^{57}$ A. C. Hindmarsh. ODEPACK, a systematized collection of ODE solvers. In R. S. Stepleman, editor, Scientific Computing, volume 1, pages 55-64. North-Holland, Amsterdam, 1983. 